\title{
Validity and reliability of haemoglobin colour scale and its comparison with clinical signs in diagnosing anaemia in pregnancy in Ahmedabad, India
}

\author{
D.V. Bala, ${ }^{7}$ S. Vyas, ${ }^{2}$ A. Shukla, ${ }^{1}$ H. Tiwari, ${ }^{1}$ G. Bhatt ${ }^{2}$ and K. Gupta ${ }^{3}$
}

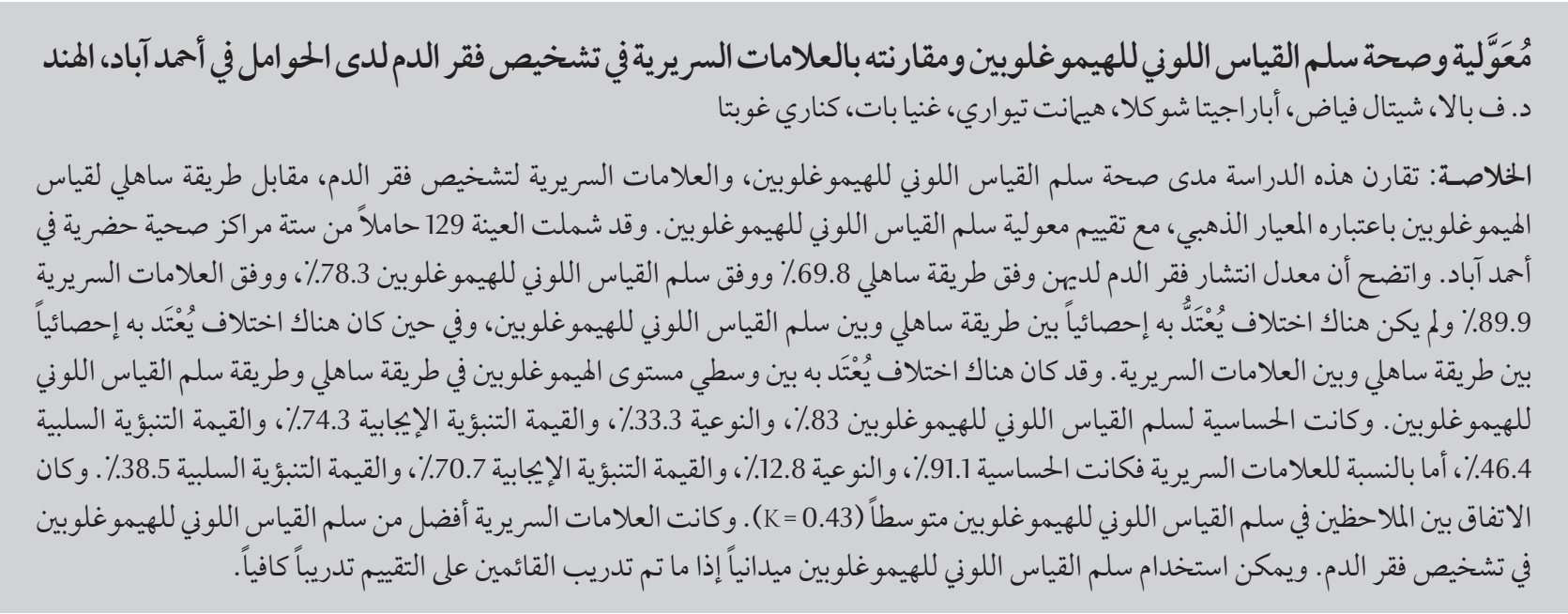

ABSTRACT This study compared the validity of the haemoglobin colour scale (HCS) and clinical signs in diagnosing anaemia against Sahli's haemoglobinometer method as the gold standard, and assessed the reliability of HCS. The sample comprised 129 pregnant women recruited from 6 urban health centres in Ahmedabad. The prevalence of anaemia was $69.8 \%$ by Sahli's method, $78.3 \%$ by HCS and $89.9 \%$ by clinical signs; there was no statistically significant difference between Sahli's method and HCS whereas there was between Sahli's method and clinical signs. The mean haemoglobin level by Sahli's method and HCS differed significantly. The sensitivity, specificity, positive predictive value and negative predictive value of HCS was $83.3 \%, 33.3 \%, 74.3 \%$ and $46.4 \%$ respectively and that of clinical signs was $91.1 \%, 12.8 \%, 70.7 \%$ and $38.5 \%$ respectively. Interobserver agreement for HCS was moderate $(\kappa=0.43)$. Clinical signs are better than HCS for diagnosing anaemia. HCS can be used in the field provided assessors are adequately trained.

Validité et fiabilité de l'échelle colorimétrique de détermination du taux d'hémoglobine et comparaison avec les signes cliniques pour le diagnostic d'anémie pendant la grossesse à Ahmedabad (Inde)

RESUMELaprésenteétudeacomparélavaliditédel'échellecolorimétriquededéterminationdutauxd'hémoglobine et les signes cliniques pour le diagnostic de l'anémie par rapport à la méthode d'hémoglobinométrie de Sahli en tant que méthode de référence, puis a évalué la fiabilité de l'échelle. L'échantillon comptait 129 femmes enceintes recrutées dans six centres de soins de santé de ville, à Ahmedabad. La prévalence de l'anémie était de 69,8\% avec la méthode de Sahli, de 78,3\% avec l'échelle colorimétrique de détermination du taux d'hémoglobine et de 89,9\% avec les signes cliniques ; aucune différence statistiquement significative n'a été observée entre la méthode de Sahli et l'échelle colorimétrique alors qu'il en existait entre la méthode de Sahli et les signes cliniques. Le taux moyen d'hémoglobine mesuré avec la méthode de Sahli et avec l'échelle colorimétrique était très différent. La sensibilité, la spécificité et les valeurs prédictives positive et négative de l'échelle colorimétrique de détermination du taux d'hémoglobine étaient de 83,3\%, 33,3\%, 74,3\% et 46,4\% respectivement et de 91,1\%, 12,8 \%, 70,7\% et $38,5 \%$ respectivement pour les signes cliniques. L'accord interobservateur pour l'échelle colorimétrique de détermination du taux d'hémoglobine était modéré $(\kappa=0,43)$. Les signes cliniques sont plus efficaces que l'échelle colorimétrique pour le diagnostic de l'anémie. L'échelle peut être utilisée sur le terrain à condition que les évaluateurs soient correctement formés. 


\section{Introduction}

Anaemia is a major public health problem globally. It affects about 1.62 billion people, which corresponds to $24.8 \%$ of the global population [1]. Women of reproductive age, particularly during pregnancy and children, especially young ones, are more vulnerable to anaemia due to their unique physiological characteristics [2,3]. Anaemia is a public health problem for pregnant women in all of the World Health Organization's (WHO) Member States [1]. In India, the prevalence of anaemia in pregnant women is $57.9 \%$ according to the National Family Health Survey 3 (NFHS 3) [4].

Given the consequences of anaemia during pregnancy, easy and accurate diagnosis is important. Haemoglobin $\mathrm{Hb})$ estimation is one simple and economic laboratory parameter to assess anaemia and is thus used quite frequently in population studies [2]. Another method used where no laboratories or few health resources are available is physical examination, which aims to identify skin or mucous membrane pallor on, for example, the conjunctiva, palms, nail beds, lips and tongue as clinical signs of anaemia $[2,3]$. Several methods are available for estimation of $\mathrm{Hb}$ in the field setting, such as the copper sulphate specific gravity method, the Lovibond comparator and portable $\mathrm{Hb}$ meters [5]. Currently Sahli's haemoglobinometer method for $\mathrm{Hb}$ estimation is the one recommended by the Government of India for use by the health workers in the field and in laboratory facilities [6].

WHO developed the haemoglobin colour scale (HCS) to screen for anaemia in the absence of laboratory-based haemoglobinometry [7]. It is a simple and inexpensive device for providing a reliable indicator of the presence and severity of anaemia [8]. Various studies have been conducted globally to validate the HCS and its sensitivity for detecting anaemia ranged from $75 \%$ to $97 \%$ while specificity ranged from $41 \%$ to $98 \%$. However, the studies had heterogeneous populations, health care settings, anaemia prevalence and findings so controversy about the usefulness of HCS still persists [9]. In this regard, it is necessary to assess diagnostic accuracy in local health care settings with local workers.

In Ahmedabad, as of June 2009, a commercial kit of HCS was provided to all the urban health centres (UHCs) to be used in the field. No validity assessment has been done so far since its introduction. Therefore, the present study was carried out to: compare the validity of HCS and that of clinical assessment against Sahli's haemoglobinometer method in diagnosing anaemia; assess the reliability of HCS; and make areaspecific recommendations in this regard.

\section{Methods}

\section{Study setting and sample}

The present study was conducted in Ahmedabad, a major city of Gujarat state in western India. The city is divided into 6 municipal zones and 57 election wards. We conducted a crosssectional study in randomly selected UHCs ( 1 per each zone of the city) during November 2009-January 2010. A list of zone-wise UHCs was obtained from the corporation office and lottery method was used to select one UHC from each zone. The study population was pregnant women attending UHCs for the first time; those already on iron therapy were excluded.

The sample size was calculated assuming a prevalence of anaemia of $58 \%$ as per NFHS 3 [4] and allowable error of $15 \%$. Women were recruited into the study as they came to the centres for an antenatal care check-up, after assessing if they met the inclusion and exclusion criteria, until the desired sample size was met from each centre (21 from 3 centres and 22 from the other 3 centres as decided by lottery method from 6 UHCs). There was no refusals to participate as $\mathrm{Hb}$ estimation is routinely done during antenatal care visits and they were informed about the study and the method. Thus a total 129 pregnant women were recruited after taking written informed consent in the vernacular language for personal interview and $\mathrm{Hb}$ estimation.

\section{Data collection}

General information was collected by the investigators by interview of each woman using a predesigned pretested questionnaire. Information was also obtained on the number of conceptions, parity, number of live children at the time of the interview and length of time between the present and previous conception.

\section{Anaemia assessment}

Assessment of anaemia by clinical signs was carried out by the medical officer of the respective UHC by identifying skin or mucous membrane pallor on conjunctiva, tongue and palms. Sahli's haemoglobinometer was also used to determine $\mathrm{Hb}$ level and was carried out by a trained laboratory technician in the respective UHC. For HCS, the commercial kit (Kruise Path, Ahmedabad) provided to all UHCs by Ahmedabad Municipal Corporation was used. The reading for HCS is taken by comparing blood drops on filter paper with colour standards. It was taken separately by 2 persons, a trained multi-purpose health worker (MPHW) or health visitor (HV) who routinely uses the HCS in the field and one of the investigators (KG).

The data so generated were used for the assessment of inter-observer variation. However, reading of the test as interpreted by MPHW/HV was used for the purpose of calculation of validity because they routinely interpret the results of HCS in the field. Blinding was carried out so that none of these individuals knew the findings of the 
others; 4 different sheets were made for recording of results by the medical officer, laboratory technician, MPHW/ $\mathrm{HV}$ and the investigator (KG). At the end of each session, these sheets were collected by another investigator for data entry. The investigator who interpreted the finding of HCS was not involved in data entry. As per WHO, $\mathrm{Hb}$ threshold for diagnosing anaemia in pregnant women is $11 \mathrm{~g} / \mathrm{dL}$ [2]. Taking this cut-off, the following classification was used:

- $\mathrm{Hb} \geq 11.0 \mathrm{~g} / \mathrm{dL}$ : no anaemia

- Hb 9.0 to $11.0 \mathrm{~g} / \mathrm{dL}$ : mild anaemia

- Hb 7.0 to $9.0 \mathrm{~g} / \mathrm{dL}$ : moderate anaemia

- $\mathrm{Hb}<7 \mathrm{~g} / \mathrm{dL}$ : severe anaemia.

\section{Ethical considerations}

Ethical clearance for the study was obtained from the Institutional Ethics Committee for Research in Human Subjects of the Smt. NHL Municipal Medical College, Ahmedabad. The women who were diagnosed as having anaemia were given iron folic acid treatment as per state guidelines.

\section{Data analysis}

Data were entered in a master chart to determine the following validity indicators:

- Sensitivity of the individual tests: defined as the ability of the test to identify correctly those who have the disease [10].

- Specificity of the individual tests: defined as the ability of the test to identify correctly those who do not have the disease [10].
- Predictive values of the individual tests [10]:

- Positive predictive value (PPV): probability of having disease in a patient with a positive test.

- Negative predictive value (NPV): probability of not having disease in a patient with a negative test.

Reliability of HCS was assessed by calculating the inter-observer variations in readings of HCS using Kappa statistics [10]. To study correlation between the Sahli and HCS methods, a Bland Altman plot was used [11].

Data analysis was done using Microsoft Excel, MedCalc, version 11.1.1.0 and SPSS, version 17.0.

\section{Results}

The mean age of the pregnant women was 24.2 [standard deviation (SD) 3.7] years and mean months of amenorrhoea was 4.71 (SD 2.1) at the time of registration.

Of the 129 women included in the study, $69.8 \%$ were diagnosed with anaemia by Sahli's method, $78.3 \%$ by HCS and $89.9 \%$ by clinical signs. There was no significant difference between the proportions diagnosed with anaemia by Sahli's method and HCS $(P=0.16)$ whereas there was a significant difference between the proportions diagnosed by Sahli's method and by clinical signs $(P=0.0001)$. With regard to the categories of anaemia, no significant difference was found between Sahli's method and HCS (all $P>0.05$ ). However, there was significant difference found in the proportion with moderate anaemia by Sahli's method and by clinical signs $(P=0.02)$ (Table 1$)$.

The mean $\mathrm{Hb}$ levels of the pregnant women by Sahli's method was 9.97 (SD 1.35) $\mathrm{g} / \mathrm{dL}$ and by HCS was 9.51 $(\mathrm{SD} 1.61) \mathrm{g} / \mathrm{dL}$, a significant difference ( $P=0.0031$, Mann-Whitney test). The Bland Altman plot (Figure 1) showed a mean difference of $0.5 \mathrm{~g} / \mathrm{dL}$ between the 2 methods with limits of agreement of $-2.7 \mathrm{~g} / \mathrm{dL}$ to $3.6 \mathrm{~g} / \mathrm{dL}$; this can be considered significant clinically because the treatment changes with change of category of anaemia, which has interval of $2 \mathrm{~g} / \mathrm{dL}$. As the mean $\mathrm{Hb}$ increased the difference between the 2 methods became smaller. For lower average $\mathrm{Hb}$ values, the HCS reading showed lower values compared to the Sahli reading, while for higher mean $\mathrm{Hb}$ values, the HCS showed higher reading. The correlation coefficient was 0.4077 (95\% confidence interval: 0.2526-0.5423) $(P<0.0001)$

The validity indicators showed that the sensitivity to differentiate anaemia from nonanaemia was higher for clinical signs (91.1\%) than for HCS (83.3\%), while specificity, PPV and NPV were higher for HCS (Table 2). In addition, the sensitivity of clinical signs to diagnose mild and moderate anaemia was higher than HCS while for severe anaemia both methods had $100 \%$ sensitivity (Table 3). Weighted kappa value for HCS was 0.43 , indicating moderate inter-observer agreement.

In $14.7 \%$ of cases, there was no difference in $\mathrm{Hb}$ readings between HCS and Sahli's method, while $55.8 \%$ of

\begin{tabular}{|c|c|c|c|c|c|}
\hline \multirow[t]{2}{*}{ Severity } & \multirow{2}{*}{$\begin{array}{c}\text { Sahli's method } \\
\%\end{array}$} & \multirow{2}{*}{$\begin{array}{c}\text { HCS } \\
\%\end{array}$} & \multirow{2}{*}{$\begin{array}{c}\text { Clinical signs } \\
\%\end{array}$} & \multicolumn{2}{|c|}{$P$-value } \\
\hline & & & & Sahli vs HCS & Sahli vs clinical signs \\
\hline Mild anaemia & 55.0 & 53.4 & 61.2 & 0.89 & 0.38 \\
\hline Moderate anaemia & 13.2 & 21.7 & 24.8 & 0.10 & 0.02 \\
\hline Severe anaemia & 1.6 & 3.2 & 3.9 & 0.66 & 0.45 \\
\hline Anaemia of any grade & 69.8 & 78.3 & 89.9 & 0.16 & 0.0001 \\
\hline
\end{tabular}

HCS = haemoglobin colour scale. 


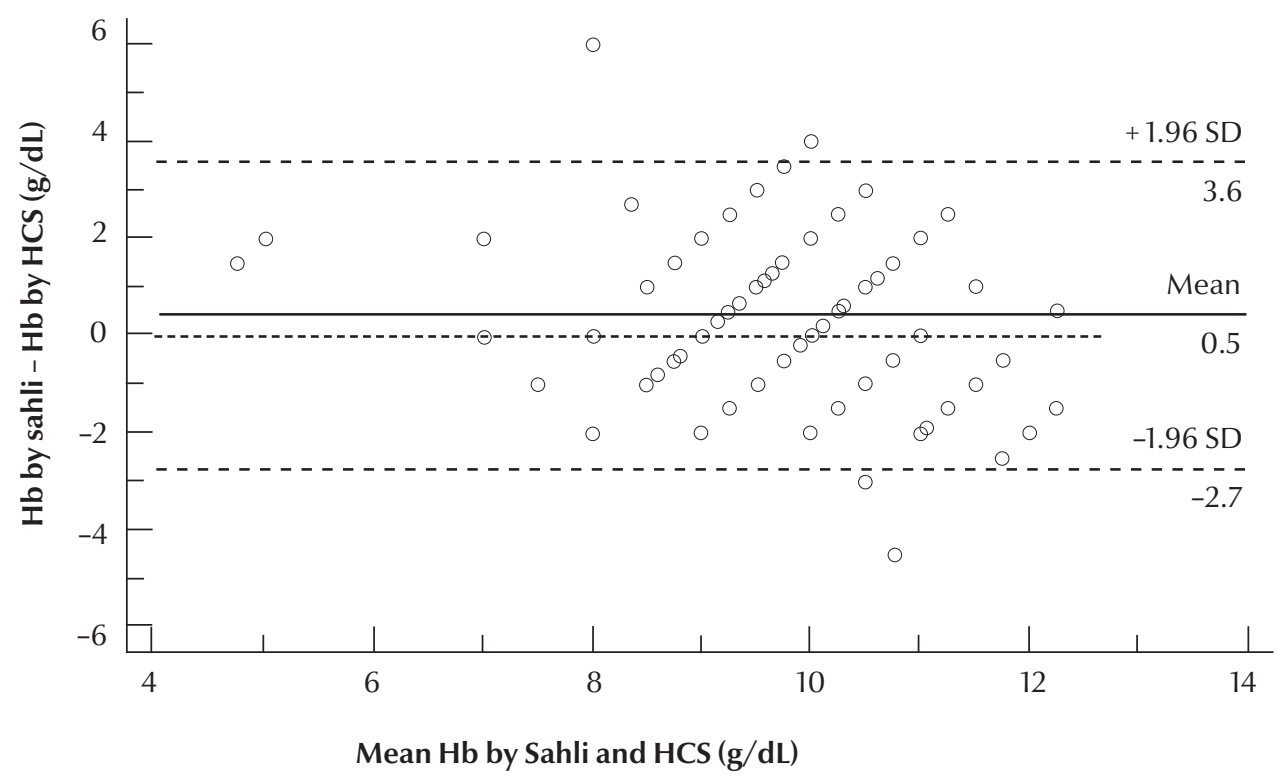

Figure 1 Bland Altman plot for haemoglobin colour scale compared with Sahli's method (SD = standard deviation)

cases, the difference was $\pm 1 \mathrm{~g} / \mathrm{dL}$, and in $82.9 \%$, the difference was within $\pm 2 \mathrm{~g} /$ $\mathrm{dL}$; in $17.1 \%$, the difference was $>2 \mathrm{~g} / \mathrm{dL}$.

\section{Discussion}

In the present study, the prevalence of anaemia in pregnant women was $69.8 \%$ which is higher than the national average according to NFHS 3 (58\% ) [4]. Various factors, such as physiological changes during pregnancy, lower socioeconomic and educational status of the women, may account for this difference. For each category of anaemia the proportion was highest by clinical assessment, which may be on account of the clinician's subjective preference or tendency chance missing any anaemic woman.
Quantitative analysis of paired observation showed that the reading of HCS was $0.46 \pm 1.63 \mathrm{~g} / \mathrm{dL}$ lower than Sahli's method which was statistically significant. A similar result was found in the Islamic Republic of Iran in a study of blood donors, with difference of $0.32 \pm 0.65 \mathrm{~g} / \mathrm{dL}$ compared with an automated cell counter [12].

The sensitivity of clinical signs in diagnosing mild and moderate anaemia was higher than that of HCS. For severe anaemia the sensitivity of both the methods was 100\%; this may be because only a few of the women were diagnosed with severe anaemia by the standard method. Other studies have reported different findings; they found that HCS was better than clinical signs for diagnosing anae$\operatorname{mia}[8,13,14]$.
In the present study the sensitivity of HCS was $83.3 \%$ and specificity $33.3 \%$. Other studies also reported HCS with sensitivities above 80\% [14-16]. However, Anand et al. found a sensitivity of $30 \%$ and specificity of $100 \%$ for HCS when compared with an autoanalyser [6]. This is the only study that we found showing such a low sensitivity. The exact reason is not clear but there were differences in the methodology which might have contributed to such differences in results, e.g. the gold standard method used in their study was an autoanalyser, the HCS kit used was from a different manufacturer and the study population taken was not vulnerable to anaemia.

Given the high prevalence of anaemia in pregnancy, the ideal instrument for diagnosis would be one with a high sensitivity and low false negative results

\begin{tabular}{lccc}
\hline \multicolumn{4}{l}{ Table 2 Validity indicators of haemoglobin colour scale and clinical signs against Sahli's method to diagnose anaemia } \\
\hline Validity indicator & Haemoglobin colour scale & Clinical signs & Simultaneous testing of both \\
Sensitivity (95\% Cl) [\%] & $83.33(74.0-90.36)$ & $91.11(83.23-96.08)$ & $96.67(90.58-99.31)$ \\
Specificity (95\% Cl) [\%] & $33.33(19.09-50.22)$ & $12.82(4.30-27.43)$ & $10.26(2.86-24.23)$ \\
PPV (95\% Cl) [\%] & $74.26(64.60-82.44)$ & $70.69(61.52-78.77)$ & $71.31(62.39-79.17)$ \\
NPV (95\% Cl) [\%] & $46.43(27.51-66.13)$ & $38.46(13.86-68.42)$ & $57.14(18.14-90.11)$ \\
Accuracy [\%] & 68.2 & 67.4 & 70.54 \\
\hline
\end{tabular}

$C I=$ confidence interval $; P P V=$ positive predictive value $N P V=$ negative predictive value . 


\begin{tabular}{|c|c|c|}
\hline \multirow{2}{*}{ Category of anaemia } & \multicolumn{2}{|c|}{ Sensitivity (95\% CI) [\%] } \\
\hline & Haemoglobin colour scale & Clinical signs \\
\hline Mild & $59.15(46.83-70.72)$ & $69.01(56.91-79.43)$ \\
\hline Moderate & 41.18 (18.43-67.04) & $47.06(22.96-72.20)$ \\
\hline Severe & $100.00(15.81-100.00)$ & $100.00(15.81-100.00)$ \\
\hline
\end{tabular}

$\mathrm{Cl}=$ confidence interval.

as an increase in false negatives would exclude some anaemic women from anaemia combat programmes in place in India, which would be harmful in places with high anaemia prevalence rates.

One of the advantages in using the HCS is that training of examiners is easy, fast and economic, and requires minimal material to distinguish between the different colour variations of the scale [7]. However, it is important to follow the instructions on how to hold the instrument and use the correct thickness of blood drop, adequate environmental luminosity and the established reading time $[7,8,17]$ and this should be emphasized in training. Similarly, training can also be given for clinical signs to the health workers as its sensitivity for identifying each category of anaemia is higher.

Comparison with otherstudiesis difficult as the gold standard method used and the study population differ in different studies. Taking into consideration financial and other resources, Sahli's method is widely used at health centres in our study area. So Sahli was taken as the gold standard in the present study although it is not considered the gold standard for $\mathrm{Hb}$ estimation. Although laboratory technicians were previously trained for Sahli's method and $\mathrm{MPHW} / \mathrm{HV}$ were trained for HCS, there might be minor differences due to subjective preferences.

As per the present study, assessment by clinical signs is a better method for diagnosing anaemia as compared to HCS. The scope of HCS seems to be limited because of its need for precise application and proper, more stringent training for the health workers is needed with further comparison with the standard method. Accuracy in both methods may be improved as examiners acquire more experience, enabling them to reduce classification errors. It is recommended that the MPHW/HV diagnose anaemia by looking for clinical signs and should start treatment on that basis; the patient can then be referred to the laboratory for confirmation and follow up. HCS can be used in cases where there is no possibility of the patient getting to a laboratory for follow up and in uncertain cases to support clinical examination.

Because detection of anaemia is important in order to prevent its adverse consequences, further studies are required to determine the effect of the various screening methods on longer term clinical outcomes, i.e. less anaemia at time of delivery among pregnant women, increased birth weight of babies, reduced maternal mortality, along with consideration of cost, rather than focussing only on diagnostic accuracy. Such studies will help health planners in choosing the cost-effective screening method for anaemia.

\section{Acknowledgements}

We thank the Medical Officer of Health, Ahmedabad Municipal Corporation for material and technical support. We also thank all the women who agreed to participate in the study and the medical officers and paramedical workers of UHCs who cooperated with us in accomplishing the study

\section{References}

1. De Benoist B et al., eds Worldwide prevalence of anaemia 1993-2005: WHO global database on anaemia. Geneva, World Health Organization, 2008 (http://whqlibdoc.who.int/publications/2008/9789241596657_eng.pdf, accessed 21 March 2012)

2. United Nations Children's Fund), United Nations University, World Health Organization. Iron deficiency anaemia. Assessment, prevention and control: a guide for programme managers. Geneva, World Health Organization, 2001 (WHO/ NHD/01.3.).

3. Demayer EM. Preventing and controlling iron deficiency anaemia through primary health care. A guide for health administrators and programme managers. Geneva, World Health Organization, 1989.
4. National Family Health Survey, India. Key indicators for India from NFHS 3 (http://www.nfhsindia.org/pdf/India.pdf, accessed 21 March 2012).

5. Stone JE et al. An evaluation of methods of screening for anemia. Bulletin of the World Health Organization, 1984, 82:115120.

6. Anand H, Mir R, Saxena R. Hemoglobin color scale a diagnostic dilemma. Indian Journal of Pathology \& Microbiology, 2009, 52:360-362.

7. Stott GJ, Lewis SM. A simple and reliable method for estimating haemoglobin. Bulletin of the World Health Organization, 1995, 73:369-373. 
8. Ingram CF, Lewis SM. Clinical use of WHO haemoglobin colour scale: Validation and critique. Journal of Clinical Pathology, 2000, 53:933-937.

9. Critchley J, Bates I. Haemoglobin colour scale for anaemia diagnosis where there is no laboratory: a systematic review. International Journal of Epidemiology, 2005, 34:1425-1434.

10. Assessing the validity and reliability of diagnostic and screening tests. In: Gordis L. Epidemiology, 5th ed. Philadelphia, WB Saunders, 2008.

11. Bland JM, Altman DG. Statistical methods for assessing agreement between measurement. Biochimica Clinica, 1987, 11:399-404.

12. Javadzadeh Shahshahani H, Amiri F. [Validity of hemoglobin color scale in blood donor screening based on Standard Operating Procedures of Iranian Blood Transfusion Organization]. The Scientific Journal of Iranian Blood Transfusion Organization, 2009, 5(4):281-286 [In Farsi].

13. Leal LP, Osório MM. Diagnostic accuracy comparison between clinical signs and hemoglobin color scale as screening methods in the diagnosis of anemia in children. Revista Brasileira de Saúde Materno Infantil, 2006, 6(2):183-189, (http:// www.scielo.br/pdf/rbsmi/v6n2/30915.pdf, accessed 20 March 2012).

14. Montresor A et al. Performance of the haemoglobin colour scale in diagnosing severe and very severe anaemia. Tropical Medicine \& International Health, 2003, 8:619-624.

15. Montresor A et al. Field trial of a haemoglobin colour scale: an effective tool to detect anaemia in preschool children. Tropical Medicine \& International Health, 2000, 5:129-133.

16. Barduagni P et al. Performance of Sahli and colour scale methods in diagnosing anaemia among school children in low prevalence areas. Tropical Medicine \& International Health, 2003, 8:615-618.

17. Gosling $\mathrm{R}$ et al. Training health workers to assess anaemia with the WHO haemoglobin colour scale. Tropical Medicine \& International Health, 2000, 5:214-221.

\section{Counselling for maternal and newborn health care: a handbook for building skills}

Counselling for maternal and newborn health care: a handbook for building skills is a practical handbook that aims to strengthen counselling and communication skills of skilled attendants (SAs) and other health providers, helping them to effectively discuss with women, families and communities the key issues surrounding pregnancy, childbirth, postpartum, postnatal and post-abortion care. The MNH Counselling Handbook is chiefly designed to be used by groups of SAs with the help of a facilitator.

The MNH counselling handbook is divided into 3 main sections. Part 1 is an introduction which describes the aims and objectives and the general layout of the handbook. Part 2 describes the counselling process and outlines the 6 key steps to effective counselling. Part 3 focuses on different maternal and newborn health topics, including general care in the home during pregnancy; birth and emergency planning; danger signs in pregnancy; post-abortion care; support during labour; postnatal care of the mother and newborn; family planning counselling; breastfeeding; women with HIV/AIDS; death and bereavement; women and violence; linking with the community.

Further information about this and other WHO publications is available at: http://www.who.int/publications/en/ 